\title{
State Flip at Exceptional Points in Atomic Spectra
}

\author{
Henri Menke, ${ }^{*}$ Marcel Klett, ${ }^{\dagger}$ Holger Cartarius, Jörg Main, and Günter Wunner \\ Institut für Theoretische Physik 1, Universität Stuttgart, 70550 Stuttgart, Germany
}

\begin{abstract}
We study the behavior of the non-adiabatic population transfer between resonances at an exceptional point in the spectrum of the hydrogen atom. It is known that, when the exceptional point is encircled, the system always ends up in the same state, independent of the initial occupation within the two-dimensional subspace spanned by the states coalescing at the exceptional point. We verify this behavior for a realistic quantum system, viz. the hydrogen atom in crossed electric and magnetic fields. It is also shown that the non-adiabatic hypothesis can be violated when resonances in the vicinity are taken into account. In addition, we study the non-adiabatic population transfer in the case of a third-order exceptional point, in which three resonances are involved.

PACS numbers: $32.60 .+\mathrm{i}, 02.30 .-\mathrm{f}, 32.80 . \mathrm{Fb}$
\end{abstract}

\section{INTRODUCTION}

In many cases a very effective way of investigating open quantum systems with reasonable effort, in particular to avoid expensive time-dependent calculations, is made possible by non-Hermitian Hamiltonians [1]. A typical example are resonances, i.e. decaying unbound states. With appropriate methods as the complex scaling approach [1-5] resonances can be uncovered in a timeindependent calculation as complex eigenvalues of the stationary Schrödinger equation. It is well known that resonances show characteristic effects not observable in Hermitian quantum systems. This is in particular true close to exceptional points $[1,6-8]$, isolated points in a physical parameter space, at which two or even more eigenstates coalesce.

The appearance of exceptional points has theoretically been shown in unstable lasers [9], optical wave guides [10] and resonators $[11,12]$. In quantum systems their existence has been proved in atomic [13-16] or molecular [17] spectra, in the scattering of particles at potential barriers [18], in atom waves [19-22], and in non-Hermitian Bose-Hubbard models [23]. Their relation to Fano resonances has been pointed out [24-26]. The experimental verification of their physical nature was achieved in microwave cavities [27-29], electronic circuits [30], metamaterials [31], a photonic crystal slab [32], and excitonpolariton resonances [33].

A simple example is the two-dimensional matrix

$$
\boldsymbol{M}(\lambda)=\left(\begin{array}{cc}
1 & \lambda \\
\lambda & -1
\end{array}\right)
$$

with a one-dimensional complex parameter $\lambda$. The eigenvalues are given by $\epsilon_{1}=\sqrt{1+\lambda^{2}}$ and $\epsilon_{2}=-\sqrt{1+\lambda^{2}}$. It is obvious that these eigenvalues share a common degeneracy for $\lambda= \pm \mathrm{i}$ and the same holds for the eigenvectors, i.e. $\lambda=\lambda_{c}= \pm \mathrm{i}$ is an exceptional point. The example

\footnotetext{
* Henri.Menke@itp1.uni-stuttgart.de

† Marcel.Klett@itp1.uni-stuttgart.de
}

demonstrates one of the most striking features of an exceptional point, which can be seen from a power series expansion of the eigenvalues for a circle $\lambda(\varphi)=\mathrm{i}+\varrho \mathrm{e}^{\mathrm{i} \varphi}$ with a small radius $\varrho$ around an exceptional point,

$$
\epsilon_{1}=\sqrt{2 \varrho} \mathrm{e}^{\mathrm{i}(\pi / 4+\varphi / 2)}, \quad \epsilon_{2}=\sqrt{2 \varrho} \mathrm{e}^{\mathrm{i}(5 \pi / 4+\varphi / 2)} .
$$

Evidently the eigenvalues interchange their position in energy space when the exceptional point is encircled in a closed loop, i.e. $\varphi=0 \ldots 2 \pi$. Only after two circles the eigenvalues return to their original positions, but the eigenvectors pick up a geometric phase, which is expressed by a sign change, e.g. $\left[\psi_{1}, \psi_{2}\right] \stackrel{\text { circle }}{\longrightarrow}\left[\psi_{2},-\psi_{1}\right]$.

This is an example of a second-order exceptional point (EP2), but also higher-order exceptional points are possible [6]. The simplest extension is a third-order exceptional point (EP3), at which three resonances coalesce, i.e. have identical eigenvalues and eigenvectors $[20,21,34-37]$.

Uzdin et al. [38] as well as Berry and Uzdin [39] have shown that the adiabatic exchange of the states mentioned above will not be observable for the true temporal evolution of an occupied resonance state. Only one of the states behaves according to the adiabatic expectation. The reason is that non-adiabatic effects can never be neglected in the decay dynamics of resonances and the adiabatic connections [40] are no longer fulfilled. When an exceptional point is encircled the occupation always ends up in the same state, independent of the initial occupation within the two-dimensional subspace of the resonances forming the exceptional point $[38,39,41-$ 44]. Recently a careful analysis of the dynamics revealed that it is strongly non-intuitive [45]. Note that the adiabatic expectation with the exchange of two resonances can always be extracted from evaluations of their complex eigenvalues if the physical parameters are changed in small steps on a closed loop in the parameter space and the eigenvalues are then connected continuously, which has been shown in numerical studies and experiments $[7,14,16,18,20,21,27-29,33]$.

It has been shown that EP2s can be exploited for the controlled occupation of a single quantum state $[42,43,46]$. However, in these considerations the two resonances coalescing at the exceptional point have always 
been assumed to be isolated from all other states. This is very often not the case in physical systems. Leclerc et al. [47] have shown that the existence of further resonances in the vicinity of an EP can significantly influence the non-adiabatic temporal evolution and the exchange behavior of states at an exceptional point.

In this paper we will address this question in detail. We do this by investigating the resonances of the hydrogen atom in crossed electric and magnetic fields. It is especially suited for this investigation since numerically exact calculations of resonance states are feasible, a large number of exceptional points is known, and their properties are clearly observable $[16,35,48]$. In particular, examples of exceptional points with additional resonances in their neighborhood are available. Some possess neighboring resonances in their close vicinity, others are very isolated. In addition, a case of two adjacent second-order exceptional points has been discovered, of which the permutation behavior of the resonances is exactly that of a third-order exceptional point if both exceptional points are encircled together. This gives us the opportunity to study the non-adiabatic state transfer at third-order exceptional points.

The remaining sections of this article are organized as follows. In Sec. II we introduce our system, viz. the hydrogen atom in crossed static electric and magnetic fields, the numerically accurate method to calculate the resonances of the system, and the evaluation of the temporal evolution of occupation probabilities of the resonances. The non-adiabatic evolution of the resonance states is then investigated in Sec. III for the case of a secondorder and a third-order exceptional point. A discussion and conclusions are given in Sec. IV.

\section{HYDROGEN ATOM IN CROSSED ELECTRIC AND MAGNETIC FIELDS}

\section{A. Resonances of the hydrogen atom}

In atomic Hartree units without relativistic corrections or finite nuclear mass effects the Hamiltonian of the hydrogen atom in crossed static electric and magnetic fields reads

$$
H=\frac{1}{2} \boldsymbol{p}^{2}-\frac{1}{r}+\frac{1}{2} \gamma L_{z}+\frac{1}{8} \gamma^{2}\left(x^{2}+y^{2}\right)+f x,
$$

where $L_{z}$ is the $z$ component of the angular momentum, and $\gamma=B / B_{0}$ and $f=F / F_{0}$, with $B_{0}=2.35 \times 10^{5} \mathrm{~T}$ and $F_{0}=5.14 \times 10^{9} \mathrm{~V} / \mathrm{cm}$, are the dimensionless field strength parameters of the magnetic and electric fields, which are oriented along the $z$ and $x$ axis, respectively. The total energy and the parity with respect to the $(x, y)$ plane are the constants of motion. The parity is the only remaining symmetry of the system and is exploited in the calculations. All subsequent studies are done in the symmetry subspace of states with even $z$ parity. To calculate the resonances of the Hamiltonian the complex rotation method is applied [1-5]. By replacing the spacial coordinates $\boldsymbol{r}$ in the Hamiltonian and the wave functions with $b^{2} \boldsymbol{r}$, where $b$ is a complex scaling parameter, we obtain a complex symmetric Hamiltonian, in which resonances appear as discrete complex energy eigenvalues. The real part of these complex eigenvalues represents the resonance energy, its imaginary part the width.

With the introduction of semiparabolic coordinates a complex symmetric matrix representation of the Schrödinger equation can be set up in an oscillator basis [49]. This leads to the generalized eigenvalue problem

$$
\boldsymbol{A}(\gamma, f) \phi=2|b|^{4} E \boldsymbol{C} \phi,
$$

where $\boldsymbol{A}(\gamma, f)$ is a complex symmetric matrix, $\boldsymbol{C}$ is a real symmetric positive definite metric, and $E$ is the complex energy eigenvalue. The appropriate normalization of the eigenvectors in the complex extended system has to be done with the c-product [1] and reads for the generalized eigenvalue problem (4), $\phi_{i} \boldsymbol{C} \phi_{j}=\delta_{i j}$.

\section{B. Temporal evolution of the occupation probabilities of the resonances}

In our scheme the field strengths $\gamma(t)$ and $f(t)$ are varied time-dependently such that closed loops are traversed in the parameter space. This results in a time-dependent matrix $\boldsymbol{A}(t)$, and thus also time-dependent resonance energies $E_{i}(t)$ and eigenstates $\phi_{i}(t)$. To study the population transfer during the traversal of a closed loop around an exceptional point we split the state by means of the spectral decomposition into these time-dependent eigenstates $\phi_{i}(t)$ of the Hamiltonian, i.e. the expansion coefficients $a_{i}(t)$ define the occupation of a resonance state $\phi_{i}(t)$ which is an eigenstate of the Hamiltonian with the current field strengths $\gamma(t)$ and $f(t)$,

$$
\psi(t)=\sum_{i} a_{i}(t) \phi_{i}(t) .
$$

This corresponds to the instantaneous basis used in [38] to study the non-adiabatic transfer in a matrix model. In the instantaneous basis the temporal evolution of the expansion coefficients following from the Schrödinger equation (4) reads

$$
\dot{a}_{i}(t)=-\mathrm{i} E(t) a_{i}(t)-\sum_{j} a_{j}(t) \phi_{i}(t) \boldsymbol{C} \dot{\phi}_{j}(t) .
$$

The dominant effect is the decay of the resonances, which leads to a fast decrease of the occupation coefficients $a_{i}$.

For a better and more intuitive interpretation of the occupation transfer during a path around an exceptional point we introduce weighted coefficients, for which the overall decay of the probability amplitude is removed. They are meant to illustrate the relative gain and loss. The weighted coefficients are denoted by a bar and are 
TABLE I. Coordinates of some exceptional points in the spectrum of the hydrogen atom in crossed magnetic $(\gamma)$ and electric $(f)$ fields. All values are given in atomic Hartree units.

\begin{tabular}{llll}
\hline \hline \multicolumn{1}{c}{$\gamma$} & \multicolumn{1}{c}{$f$} & $\operatorname{Re}(E)$ & $\operatorname{Im}(E)$ \\
0.005388 & 0.0002619 & -0.02360 & -0.00015 \\
0.00611 & 0.000256 & -0.01593 & -0.00024 \\
0.00615 & 0.000265 & -0.0158 & -0.000374 \\
\hline \hline
\end{tabular}

given by

$$
\bar{a}_{i}=\left|a_{i}\right|^{2}\left(\sum_{j=1}^{N}\left|a_{j}\right|^{2}\right)^{-1},
$$

where $N$ is the total number of states taken into account.

If there were no couplings between the eigenstates, i.e. $\phi_{i} \boldsymbol{C} \dot{\phi}_{j}=0$, all populations would evolve independently and only the decay of the resonances with a timedependent decay rate $\operatorname{Im}\left(E_{i}(t)\right)$ would be observed. This adiabatic expectation can be formulated as

$$
\dot{a}_{\text {ad }}(t)=-\mathrm{i} E(t) a_{\mathrm{ad}}(t) .
$$

We use it to compare the full temporal evolution given by Eq. (6) with the adiabatic approximation.

\section{STATE EXCHANGE FOR CIRCLES AROUND EXCEPTIONAL POINTS}

\section{A. Second-order exceptional point}

In the following we study the population transfer at previously determined exceptional points [35]. First we observe the behavior at a second-order exceptional point, of which the physical parameters are given in the first row of Table I. To encircle the exceptional point the parameters $\gamma$ and $f$ have to be varied in a specific way. They need to perform a closed loop, hence a good choice is a circle described by

$$
\gamma(\varphi)=\gamma_{0}(1+\delta \cos \varphi), \quad f(\varphi)=f_{0}(1+\delta \sin \varphi)
$$

where the pair $\left(\gamma_{0}, f_{0}\right)$ represents the circle's center and $\delta$ is a radius chosen relative to the field strengths. The trajectories of the resonances in energy space for a relative radius $\delta=10^{-2}$ are depicted in Fig. 1(a). The center was chosen exactly at the exceptional point. The plot shows two resonances interchanging their position during a traversal of the loop in the parameter space, which is shown in the inset. Two other resonances with a smaller modulus of the imaginary part are plotted alongside. There are even more resonances in the vicinity with greater moduli of the imaginary parts. Calculations taking these also into account were carried out, and it turned out that they do not influence the result.

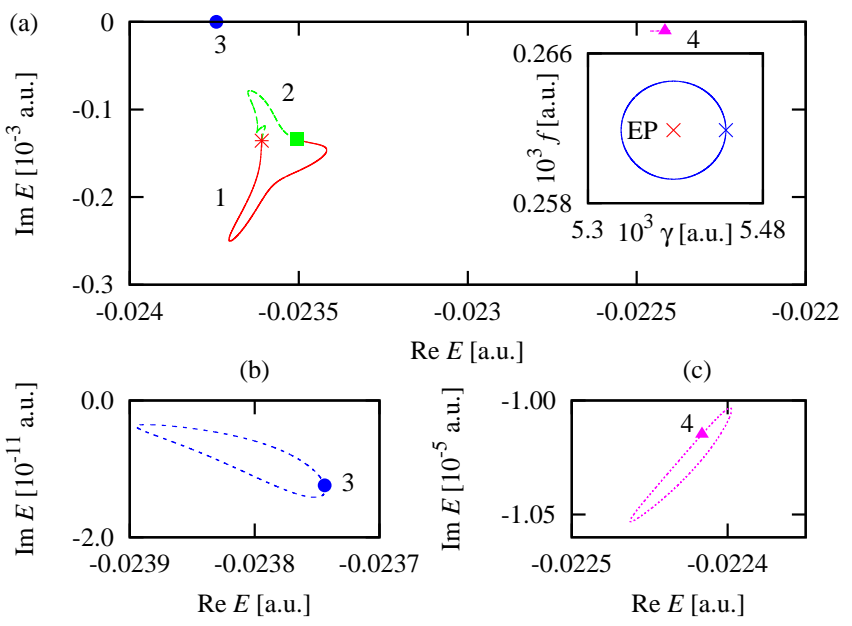

FIG. 1. (Color online) (a) Two resonances interchanging their positions in the complex energy plane for a parameter space circle (inset) around the exceptional point listed in the first line of Table I. The center of the parameter space circle is identical with the exceptional point and $\delta=10^{-2}$ was chosen. The initial point in the parameter plane and the corresponding energy values of the resonances are marked by symbols on the lines. The plots (b) and (c) show the dynamics of two nearby resonances with small imaginary parts, which are only visible as dots in (a).

We proceed to investigate how well the statement given in Uzdin et al. [38], viz. that the final distribution of the populations is independent of the initial state, is visible in the case of the hydrogen atom with its large number of highly coupled states. Therefore we prepare the system with the full population starting in one of the two resonances belonging to the exceptional point. In a first step we neglect all further states, and thus effectively reduce the Hilbert space to two dimensions. In Fig. 2 the evolution is displayed. The line styles of the respective coefficients $a_{i}$ (excluding $a_{\text {ad }}$, of course) correspond to those shown in the map in Fig. 1. We find that the adiabatic hypothesis is perfectly met for the case of the population being fully prepared in $a_{2}$, which can be graphically verified in Fig. 2(b). The final state is in both cases a majority population of the state labeled $a_{2}$ and a minority in that identified with $a_{1}$.

However, this behavior changes completely if we take into account the resonances with smaller moduli of the imaginary parts in the vicinity of the two states. An example is depicted in Fig. 3, where all four resonances shown in Fig. 1 have been used for the calculation of the temporal evolution. Due to non-adiabatic couplings between all four resonances the states represented by $a_{3}$ and $a_{4}$ gain in population even though no population was initially prepared in them. It is even more surprising that resonance $a_{4}$ dominates in the end. In principle one would expect the majority to end up in the resonance $a_{3}$ as this is a nearly bound state with the lowest imaginary part. This is indeed what is going to happen, but not 

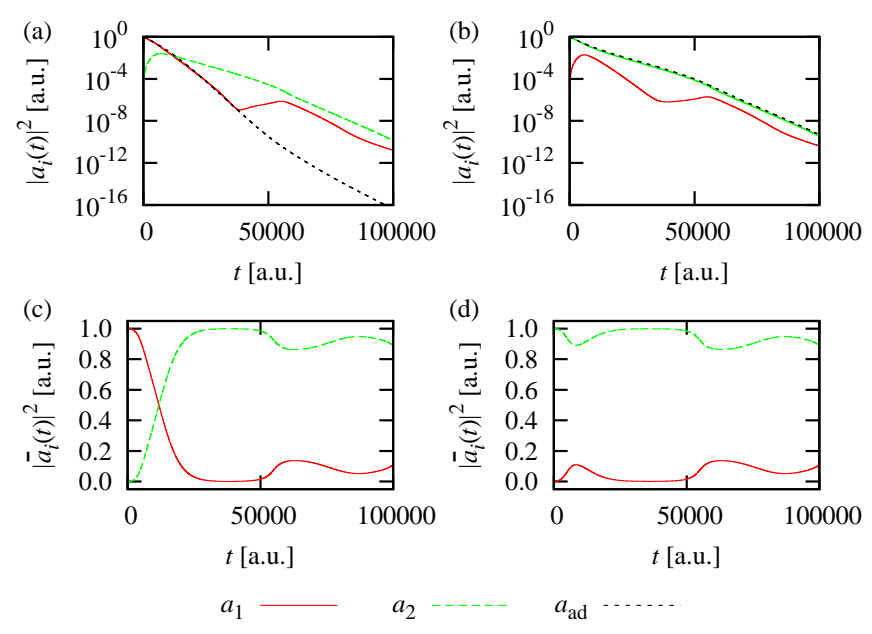

FIG. 2. (Color online) Temporal evolution of the populations. Only the two resonances connected with the exceptional point were taken into account. In (a) and (b) the actual populations are plotted for an initial population $a_{1}(0)=1$ and $a_{2}(0)=1$, respectively. The overall evolution is dominated by the decay emergent from the non-zero imaginary parts of the eigenvalues. The weighted coefficients according to Eq. (7) are shown for the initial condition of (a) in (c) and for that of (b) in (d). The results of the adiabatic approximation $\left(a_{\mathrm{ad}}\right)$ are shown for comparison.

on the time scales we used in the calculations. Since the time is not sufficient for the occupation in $a_{4}$ to vanish, the stronger coupling of that state to $a_{1}$ and $a_{2}$ decides on the final population. The important statement holds in spite of this, viz. that the populations of the states performing the exchange vanish while the populations of the states with small imaginary parts persist.

\section{B. Importance of exceptional points for the exchange behavior}

We have seen that the decay rates, i.e. the imaginary parts of the complex energy eigenvalues, basically determine which nearby resonance of an initially occupied state survives at the end of the parameter space loop. This raises the question whether the exceptional point really is important for the exchange behavior since nonadiabatic couplings and strongly unequal decay rates can appear without the existence of exceptional points for any pair of resonances with similar energies as well. To address this question we move the center of the parameter space circle as shown in Fig. 4(a). The first circle is, as before, exactly centered at the exceptional point. Then it is shifted in small steps to larger values of $\gamma$ via the shift parameter $s$. The modified circle reads

$$
\gamma(\varphi)=\gamma_{0}[1+\delta(s+\cos \varphi)], \quad f(\varphi)=f_{0}(1+\delta \sin \varphi) .
$$

To get an intuitive insight we reduce the calculation again to the two resonances involved in the exceptional point.
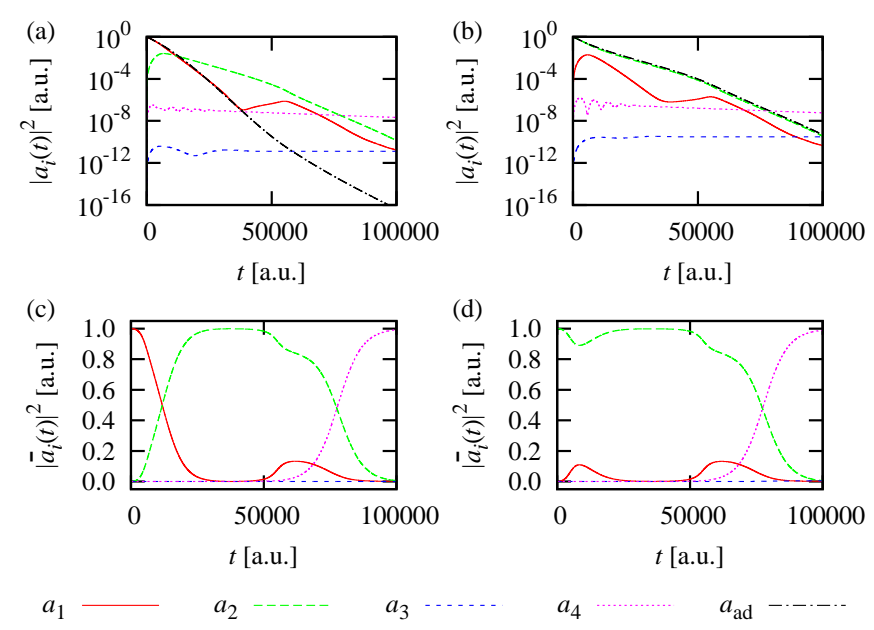

FIG. 3. (Color online) Temporal evolution of the populations with all four resonances taken into account. In (a) the initial population was exclusively in the resonance described by the coefficient $a_{1}$ and in (b) only $a_{2}$ was initially populated. In (a) and (b) it is already visible that the resonances with smaller moduli of the imaginary parts decay much more slowly. Eventually the resonance with a smaller modulus of the imaginary part dominates. This is especially well visible in (c) and (d), in which the weighted coefficients for the cases of (a) and (b), respectively, are shown. The whole surviving population transfers into the resonance represented by $a_{4}$.

This removes the overall effect of the unavoidable transition to the slowest decaying nearby resonance and helps us to focus on the effect of the exceptional point.

The temporal evolution of the resonances for this case can be seen in Fig. 4(b) and the representation with weighted coefficients is given in Fig. 4(c). In all cases the initial population was exclusively in the state labeled with the coefficient $a_{1}$. This is the non-adiabatic case from above. As long as the exceptional point is located inside the parameter space loop there are only slight changes of the temporal evolution. In particular, the final majority population of the coefficient $a_{2}$ remains unchanged. As soon as the exceptional point lies outside the parameter space circle the evolution of the occupations changes suddenly. One can observe that for $s=1.1$ the dominating population is in the coefficient $a_{1}$, a result which agrees with the adiabatic expectation. Since there is no longer a permutation of the resonances even in the purely adiabatic case if the exceptional point is not encircled, the switch of the majority population from one state to the other is not very surprising. However, the influence of the exceptional point is even stronger. It is additionally expressed in the total difference of the occupation of both states. If an exceptional point is encircled, it is much more pronounced as in the case in which there is no exceptional point within the circle.

For an even stronger shift of the circle the difference in the final population of $a_{1}$ and $a_{2}$ is reduced further and the majority population switches again. This happens 

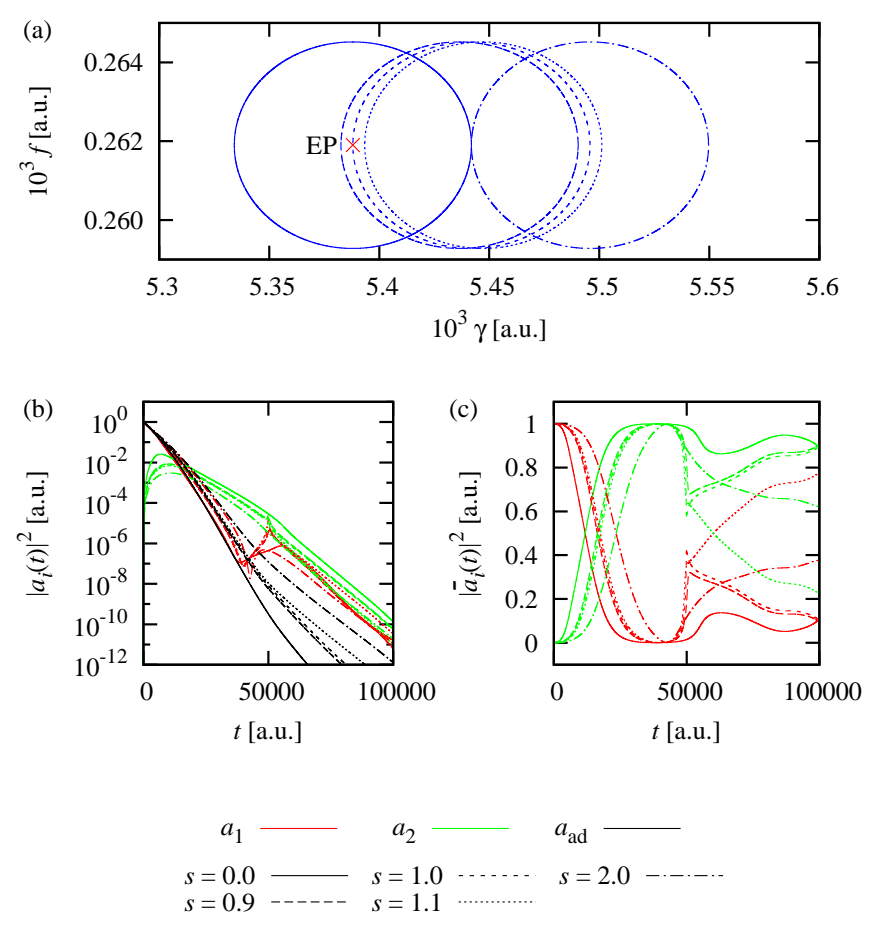

FIG. 4. (Color online) The center of the circle in parameter space is shifted in positive $\gamma$ direction to study the transition from an interchange scenario to a non-interchange case. (a) Circles for different values of $s$ as introduced in Eq. (10). The time evolution of the absolute (b) and weighted (c) coefficients demonstrate a significant qualitative change as soon as the exceptional point is no longer located within the parameter space loop. The line styles of the circles correspond to those used in the temporal evolution. For the non-weighted coefficients the adiabatic expectation is plotted alongside.

in a smooth way and can be traced back to the different decay rates.

The whole scenario can be understood even better with Fig. 5, in which the final population of both resonances is shown in dependence on the shift parameter $s$. The dramatic influence of the exceptional point becomes immediately clear due to the sudden jump of both populations at the value $s=1$, for which the parameter space circle crosses the exceptional point. Thus, the total behavior uncovered in Figs. 2 and 3 cannot be explained by the non-adiabatic couplings and the different decay rates alone. The presence of the exceptional point is essential.

The relevance of the exceptional point also becomes clear in Fig. 6, in which the two weighted coefficients are plotted in dependency of the shift parameter $s$ and the evolved time $t$. It can be seen that those paths which encircle the exceptional point lead to a dramatic change in the occupation probabilities. For values $s \gtrsim 1$, i.e. close to the exceptional point, the switch from one Riemann surface to the other becomes observable. However, for larger shifts $s \approx 2$ the non-adiabatic processes lead to a change of the final result.
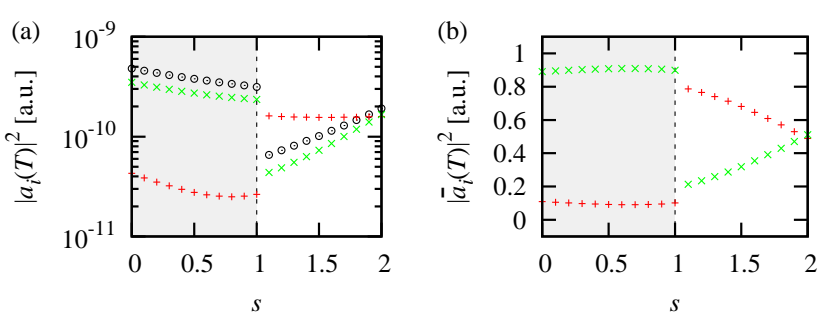

FIG. 5. (Color online) Final values of the coefficients after a full traversal of the circle in parameter space as a function of the shift parameter $s$, where the absolute coefficients (a) and their weighted counterparts (b) are shown. Red plus symbols represent $a_{1}$, green crosses stand for $a_{2}$, and the black circles mark the adiabatic approximation. The shaded area denotes that the circle for this value of $s$ encircles the exceptional point.
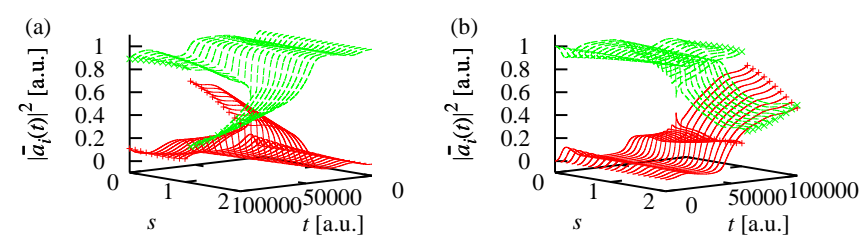

FIG. 6. (Color online) Weighted coefficients $\left|a_{i}\right|^{2}$ from Fig. 5 as a function of the shift parameter $s$ and the evolved time $t$. Two different views on the $(s, t)$ plane are shown in (a) and (b). One clearly recognizes the dramatic change as soon as the exceptional point is within the parameter space circle indicating the switch from one Riemann surface to the other.

\section{Third-order exceptional point}

The hydrogen atom does not only possess second-order exceptional points. A structure identical to that of a third-order exceptional point has also been detected [16]. It is uncovered by encircling the two exceptional points in the last two rows of Table I at once. An energy map of the resulting scheme of interchange is depicted in Fig. 7 for $\gamma_{0}=0.00609, f_{0}=0.000261$, and a radius of $\delta=$ $3.0 \times 10^{-2}$. One can see that all three resonances are permuted. In this case a closed loop of a single resonance in the complex energy plane is only achieved by three circles in the parameter space.

As in the case of the second-order exceptional point we first perform calculations which only take the three resonances connected to the EP3 structure into account. This allows us to study the non-adiabatic temporal evolution for an unperturbed EP3. For each calculation we prepare the initial population fully in one of these states. The results are plotted in Fig. 8. To facilitate the comparison the line styles correspond to those in the map of Fig. 7. The plots 8(a) and (d) correspond to the system being initially prepared in the state $a_{1}$. Until about half way through the circle in parameter space the system evolves adiabatically as can be seen in Fig. 8(a), 


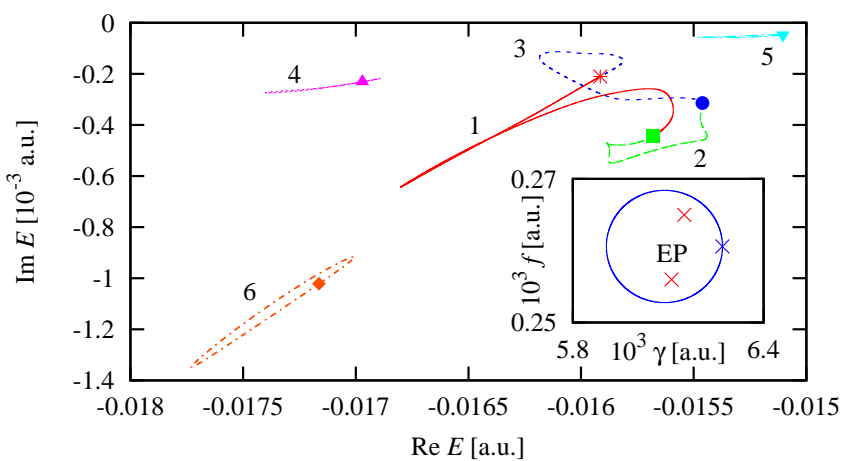

FIG. 7. (Color online) Map in the energy space of a structure identical to that of a third-order exceptional point for a parameter space circle around the two exceptional points from the last two rows of Table I (see inset). The plot shows three resonances interchanging their position and some nearby resonances in the energy space. The energy eigenvalues for the initial point on the parameter space circle are marked by symbols on the line.

i.e. the line of $a_{1}$ is exactly on top of that for the adiabatic case. However, then the lines separate and the state associated with $a_{3}$ exceeds the $a_{1}$ curve in amplitude. Fig. 8(d) shows essentially the same, but for the coefficients weighted according to Eq. (7). From this we find that the population of the state associated with $a_{1}$ is transferred into the state associated with $a_{3}$, whereas the state with $a_{2}$ is not involved at all in the population transfer.

Figures 8(b) and (e) depict the situation for the initial population being prepared in $a_{2}$. Even though there is some transition to $a_{1}$ at first, the coefficient $a_{3}$ soon dominates. After $a_{3}$ ascends to the population leader, $a_{2}$ does no longer contribute to the population. If the system is prepared with the initial population in $a_{3}$ it evolves adiabatically until the end of the present cycle time [cf. Figs. 8(c) and (f)]. As was observed above for the other cases the system also starts off adiabatically, but eventually deviates from this behavior. Hence for the initial population in $a_{1}$ or $a_{2}$ a state flip occurs. This is particularly visible in the weighted coefficients in Figs. 8(c)-(f). With these results we can extend the statement given in Uzdin et al. [38] to the scenario of three permuting resonances. Also in this case the final distribution of the populations is independent of the initial state. In the example considered it always ends up in $a_{3}$.

Of course, the three resonances forming the EP3 are not isolated in the spectra of the hydrogen atom as we observed already for the EP2. Here the closest three resonances have to be taken into account to obtain a realistic temporal evolution. All of them are included in Fig. 7. Considering the imaginary parts of the resonances during the whole parameter space loop we have one which is strictly larger, one which is strictly smaller, and one that lies somewhere in between that of the interchanging resonances. The time evolution is depicted in Fig. 9 in the same way as in Fig. 8.

Starting from the left Fig. 9(a) corresponds to the initial population being prepared fully in the state represented by $a_{1}$. Here, the system evolves adiabatically at first, but eventually $a_{5}$ exceeds all others in terms of magnitude. This is even more visible in the plot for the weighted coefficients shown in Fig. $9(\mathrm{~d})$, where $a_{5}$ rapidly approaches unity after about a quarter of the traversal time and then does not change for the rest of the evolution. A similar behavior is observed for an initial population of only the state represented by $a_{2}$, though $a_{5}$ approaches unity even faster in the weighted representation shown in Fig. 9(e). While the population prepared fully in the state corresponding to $a_{3}$ evolved adiabatically when nearby resonances were neglected, we again observe a transition into $a_{5}$ in the extended case as can be seen in Figs. 9(c) and (f).

We conjecture that the transition from all other resonances to $a_{5}$ is induced by non-adiabatic couplings. This is certainly an effect of the close distance between the resonance of $a_{5}$ and the interchanging resonances in energy space, cf. Fig. 7. One could now claim that the other resonances taken into account are also in a close distance and should acquire a considerable occupation during the traversal of the loop. However, this is not the case. They are even invisible in the diagram for the weighted coefficients. This happens due to the fact that their imaginary parts are substantially larger than that of the resonance belonging to $a_{5}$, which results in a faster decay of their population.

\section{DISCUSSION AND CONCLUSIONS}

In summary we were able to show that the nonadiabatic state flip at an EP2 is also observable in the temporal evolution of occupied resonances of the hydrogen atom in crossed electric and magnetic fields. If only the two resonances connected to an exceptional point are taken into account the system always ends up in the same state independent of the initial condition as in all previous studies $[38,39,42-45,47]$ if a parameter space loop around the exceptional point is performed. However, the spectra of the hydrogen atom always exhibit further resonances in the vicinity of those forming the exceptional point, which can drastically influence the final occupation [47]. A coupling to these states cannot be neglected and eventually the state with the smallest decay rate dominates. This could be verified in numerically exact calculations for the hydrogen atom.

Even though the non-adiabatic couplings in combination with different decay rates basically decide which resonance is occupied after a traversal of a parameter space loop they are not the only relevant information. The temporal evolution is strongly influenced by the presence of an exceptional point. If it is located within the parameter space loop the difference in the final occupation is exchanged and increases drastically. 

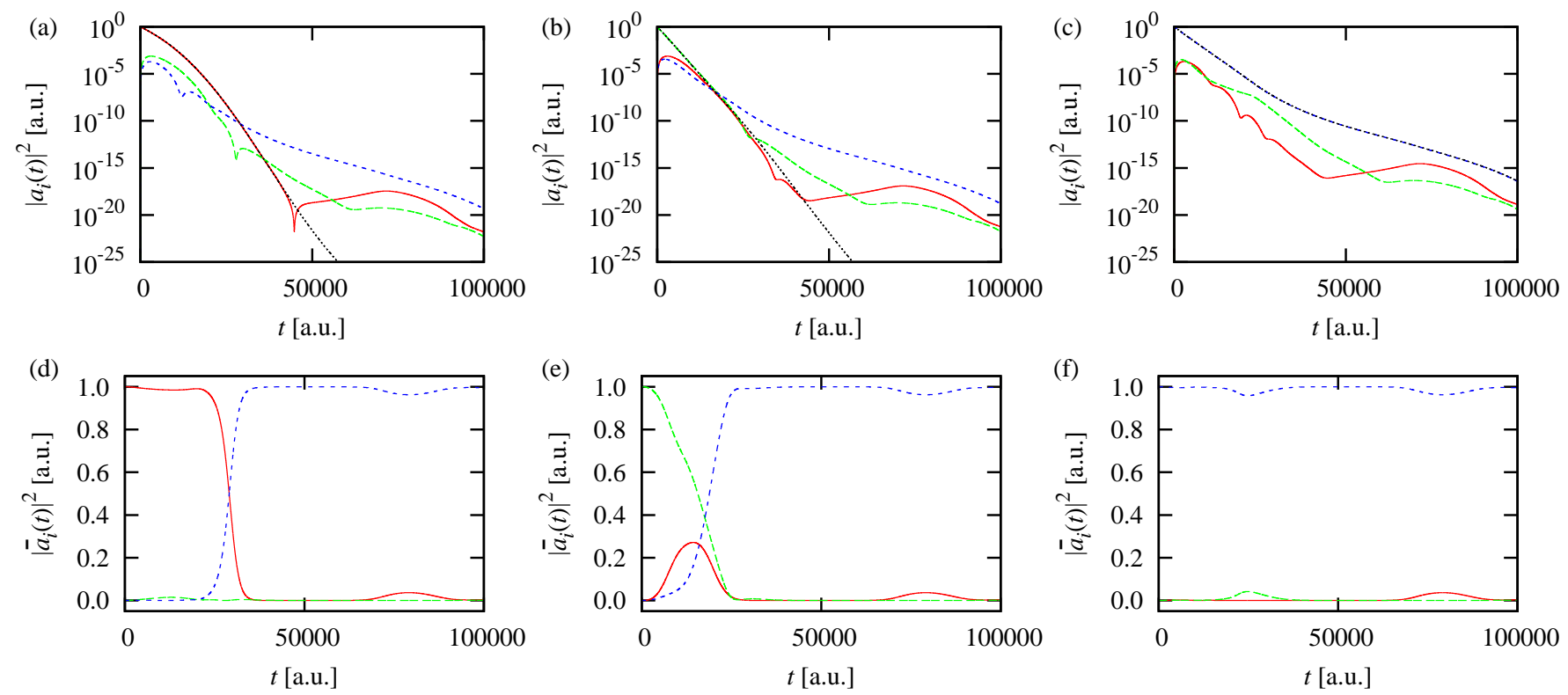

$a_{1} \longrightarrow \quad a_{2} \ldots-\ldots, a_{3} \ldots \ldots \ldots \ldots$

FIG. 8. (Color online) Temporal evolution of the populations for a circle around a third-order exceptional point. The initial populations were set up in $a_{1}\left[(\mathrm{a})\right.$ and (d)], $a_{2}\left[(\mathrm{~b})\right.$ and (e)], and $a_{3}[(\mathrm{c})$ and (f)]. In the case of the initial population being prepared in $a_{3}$ the system evolves adiabatically. In all cases the non-dissipated population ends up in $a_{3}$. This can be clearly seen in the weighted coefficients $\bar{a}_{i}$.
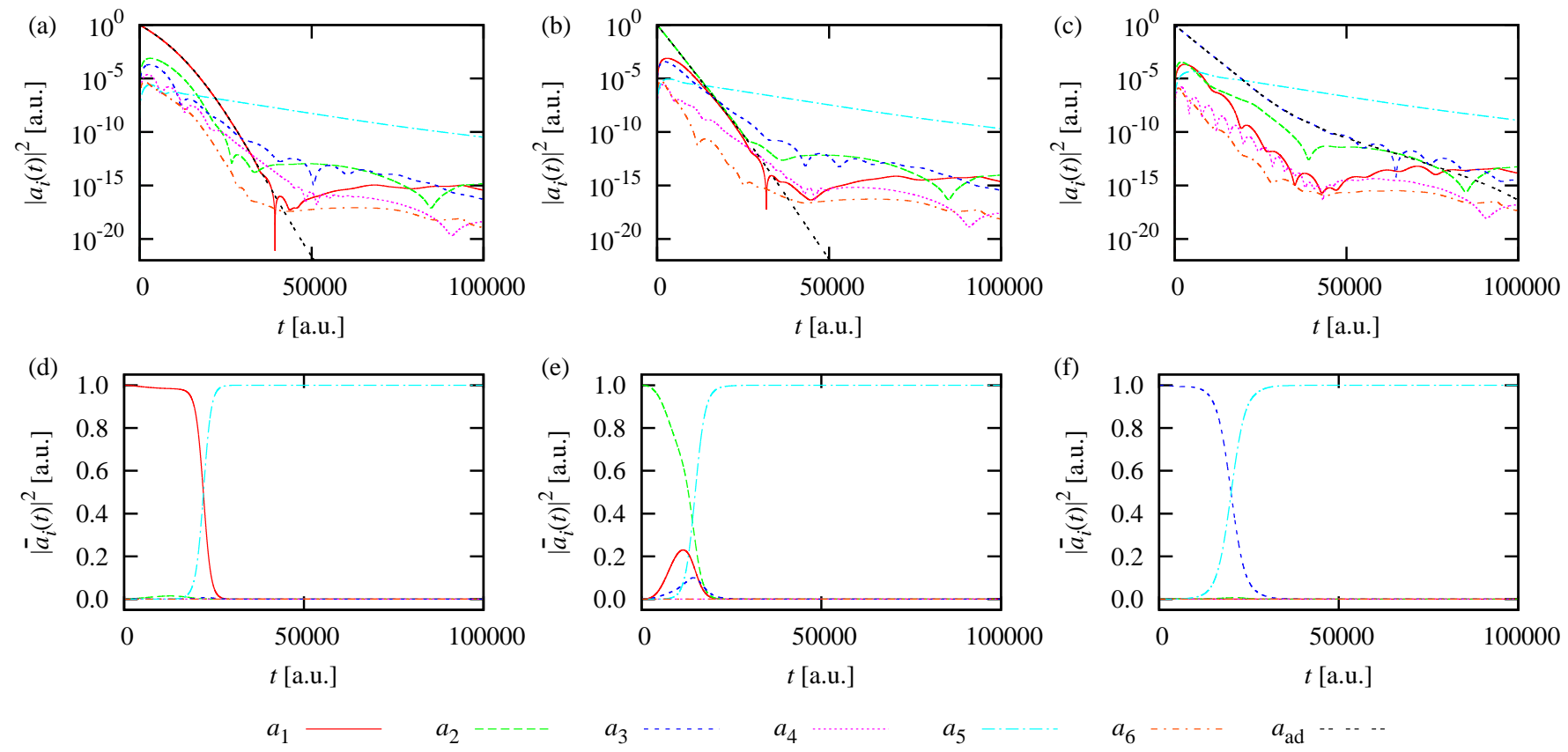

FIG. 9. (Color online) Temporal evolution of the populations for the same parameter space circle as in Fig. 8 but with six resonances taken into account in the calculation. Again, the initial populations were set up in $a_{1}\left[(\mathrm{a})\right.$ and (d)], $a_{2}[(\mathrm{~b})$ and (e)], and $a_{3}\left[(\mathrm{c})\right.$ and (f)]. The population always ends up in the resonance with the least modulus of the imaginary part, here $a_{5}$. 
Similar relations hold for third-order exceptional points. If couplings to further resonances can be neglected and the traversal time is long enough the final population always ends up in the same state and does not depend on the initial population of the three states forming the EP3. As in the EP2 case for a realistic scenario in an atomic system further resonances have to be considered and lead to a change of the behavior in favor of an exclusive population of a nearby resonance in energy space with the lowest decay rate.

The calculations reported in this work clearly show that an observation of the characteristic non-adiabatic population transfer at exceptional points will only be possible if sufficiently isolated resonances are accessible. In an atomic or molecular quantum system this will be a big challenge. For the hydrogen atom we have to remark that the parameter range we used in the calcula- tions was chosen due to the numerical capabilities and includes a basis with approximately 10000 states. This makes accessible relatively low lying energies which have to be influenced by strong fields (magnetic field $\approx 1000 \mathrm{~T}$, electric field $\approx 10^{6} \mathrm{~V} / \mathrm{cm}$ ) resulting in short decay times. Even though extremely low surviving probabilities were accepted in the calculations the traversal time of the parameter space loop is on the order of $10^{-11} \mathrm{~s}$. In an experiment this could be overcome by aiming at resonances states at higher energies, which additionally increases the probability of the appearance of exceptional points since the density of states is higher, or by investigating an almost equivalent system. Hydrogen-like excitons in semiconductor structures would lower the physical parameters to accessible values and could be studied in experiments [50].
[1] N. Moiseyev, Non-Hermitian Quantum Mechanics (Cambridge University Press, Cambridge, 2011).

[2] W. P. Reinhardt, Annu. Rev. Phys. Chem. 33, 223 (1982).

[3] N. Moiseyev, Phys. Rep. 302, 212 (1998).

[4] D. Delande, A. Bommier, and J. C. Gay, Phys. Rev. Lett. 66, 141 (1991).

[5] Y. Ho, Phys. Rep. 99, 1 (1983).

[6] W. D. Heiss, J. Phys. A 45, 444016 (2012).

[7] W. D. Heiss, Eur. Phys. J. D 7, 1 (1999).

[8] T. Kato, Perturbation Theory for Linear Operators (Springer, New York, 1966).

[9] M. V. Berry, J. Mod. Opt. 50, 63 (2003).

[10] S. Klaiman, U. Günther, and N. Moiseyev, Phys. Rev. Lett. 101, 080402 (2008).

[11] J. Wiersig, S. W. Kim, and M. Hentschel, Phys. Rev. A 78, 053809 (2008).

[12] J. Wiersig, Phys. Rev. Lett. 112, 203901 (2014).

[13] A. I. Magunov, I. Rotter, and S. I. Strakhova, J. Phys. B 32, 1489 (1999).

[14] A. I. Magunov, I. Rotter, and S. I. Strakhova, J. Phys. B 34, 29 (2001).

[15] O. Latinne, N. J. Kylstra, M. Dörr, J. Purvis, M. TeraoDunseath, C. J. Joachain, P. G. Burke, and C. J. Noble, Phys. Rev. Lett. 74, 46 (1995).

[16] H. Cartarius, J. Main, and G. Wunner, Phys. Rev. Lett. 99, 173003 (2007).

[17] R. Lefebvre, O. Atabek, M. Šindelka, and N. Moiseyev, Phys. Rev. Lett. 103, 123003 (2009).

[18] E. Hernández, A. Jáuregui, and A. Mondragán, J. Phys. A 39, 10087 (2006).

[19] K. Rapedius, C. Elsen, D. Witthaut, S. Wimberger, and H. J. Korsch, Phys. Rev. A 82, 063601 (2010).

[20] H. Cartarius, J. Main, and G. Wunner, Phys. Rev. A 77, 013618 (2008).

[21] R. Gutöhrlein, J. Main, H. Cartarius, and G. Wunner, J. Phys. A 46, 305001 (2013).

[22] N. Abt, H. Cartarius, and G. Wunner, Int. J. Theor. Phys. 54, 4054 (2015).

[23] E. M. Graefe, U. Günther, H. J. Korsch, and A. E. Niederle, J. Phys. A 41, 255206 (2008).
[24] A. I. Magunov, I. Rotter, and S. I. Strakhova, Phys. Rev. B 68, 245305 (2003).

[25] W. D. Heiss and G. Wunner, Eur. Phys. J. D 68, 284 (2014).

[26] L. Schwarz, H. Cartarius, G. Wunner, W. D. Heiss, and J. Main, Eur. Phys. J. D 69, 196 (2015).

[27] C. Dembowski, H.-D. Gräf, H. L. Harney, A. Heine, W. D. Heiss, H. Rehfeld, and A. Richter, Phys. Rev. Lett. 86, 787 (2001).

[28] B. Dietz, H. L. Harney, O. N. Kirillov, M. Miski-Oglu, A. Richter, and F. Schäfer, Phys. Rev. Lett. 106, 150403 (2011).

[29] S. Bittner, B. Dietz, H. L. Harney, M. Miski-Oglu, A. Richter, and F. Schäfer, Phys. Rev. E 89, 032909 (2014).

[30] T. Stehmann, W. D. Heiss, and F. G. Scholtz, J. Phys. A 37, 7813 (2004).

[31] M. Lawrence, N. Xu, X. Zhang, L. Cong, J. Han, W. Zhang, and S. Zhang, Phys. Rev. Lett. 113, 093901 (2014).

[32] B. Zhen, C. W. Hsu, Y. Igarashi, L. Lu, I. Kaminer, A. Pick, S.-L. Chua, J. D. Joannopoulos, and M. Soljacic, Nature 525, 354 (2015).

[33] T. Gao, E. Estrecho, K. Y. Bliokh, T. C. H. Liew, M. D. Fraser, S. Brodbeck, M. Kamp, C. Schneider, S. Höfling, Y. Yamamoto, F. Nori, Y. S. Kivshar, A. G. Truscott, R. G. Dall, and E. A. Ostrovskaya, Nature 526, 554 (2015).

[34] W. D. Heiss, J. Phys. A 41, 244010 (2008).

[35] H. Cartarius, J. Main, and G. Wunner, Phys. Rev. A 79, 053408 (2009).

[36] G. Demange and E.-M. Graefe, J. Phys. A 45, 025303 (2012).

[37] H. Eleuch and I. Rotter, Eur. Phys. J. D 69, 230 (2015).

[38] R. Uzdin, A. Mailybaev, and N. Moiseyev, J. Phys. A 44, 435302 (2011).

[39] M. V. Berry and R. Uzdin, J. Phys. A 44, 435303 (2011).

[40] V. Arnold, Sel. Math. 1, 1 (1995).

[41] I. Gilary and N. Moiseyev, J. Phys. B 45, 051002 (2012).

[42] I. Gilary, A. A. Mailybaev, and N. Moiseyev, Phys. Rev. A 88, $010102(2013)$. 
[43] E.-M. Graefe, A. A. Mailybaev, and N. Moiseyev, Phys. Rev. A 88, 033842 (2013).

[44] D. Viennot, J. Phys. A 47, 065302 (2014).

[45] T. J. Milburn, J. Doppler, C. A. Holmes, S. Portolan, S. Rotter, and P. Rabl, Phys. Rev. A 92, 052124 (2015).

[46] R. Lefebvre, O. Atabek, M. Šindelka, and N. Moiseyev, Phys. Rev. Lett. 103, 123003 (2009).
[47] A. Leclerc, G. Jolicard, and J. P. Killingbeck, J. Phys. B 46, 145503 (2013).

[48] H. Cartarius and N. Moiseyev, Phys. Rev. A 84, 013419 (2011).

[49] J. Main and G. Wunner, J. Phys. B 27, 2835 (1994).

[50] T. Kazimierczuk, D. Fröhlich, S. Scheel, H. Stolz, and M. Bayer, Nature 514, 343 (2014). 